\title{
Checkpoint inhibitor therapy in preclinical sepsis models: a systematic review and meta-analysis
}

\author{
Lindsay M. Busch ${ }^{*}$ D, Junfeng Sun, Xizhong Cui, Peter Q. Eichacker and Parizad Torabi-Parizi
}

\author{
* Correspondence: Lindsay.busch@ \\ nih.gov \\ Critical Care Medicine Department, \\ National Institutes of Health Clinical \\ Center, Bethesda, USA
}

\begin{abstract}
Background: Animal studies reporting immune checkpoint inhibitors (CPIs) improved host defense and survival during bacterial sepsis provided one basis for phase I CPI sepsis trials. We performed a systematic review and meta-analysis examining the benefit of $\mathrm{CPI}$ therapy in preclinical studies, and whether variables potentially altering this clinical benefit were investigated. Studies were analyzed that compared survival following bacteria or lipopolysaccharide challenge in animals treated with inhibitors to programmed death-1 (PD-1), PD-ligand1 (PD-L1), cytotoxic T lymphocyte-associated protein-4 (CTLA-4), or B- and T-lymphocyte attenuator (BTLA) versus control.

Results: Nineteen experiments from 11 studies $(n=709)$ were included. All experiments were in mice, and 10 of the 19 were published from a single research group. Sample size calculations and randomization were not reported in any studies, and blinding procedures were reported in just 1. Across all 19 experiments, CPIs increased the odds ratio for survival $(\mathrm{OR}, 95 \% \mathrm{Cl})[3.37(1.55,7.31)]$ but with heterogeneity $\left(I^{2}=59 \%, p<0.01\right)$. After stratification by checkpoint molecule targeted, challenge site or type, or concurrent antibacterial treatment, CPIs had consistent effects over most experiments in the 9 that included antibacterial treatment $\left[\mathrm{OR}=2.82(1.60,4.98), P^{2}=6 \%, p=0.39\right.$ with versus $4.01(0.89,18.05), P^{2}=$ $74 \%, p<0.01$ without]. All 9 antibiotic experiments employed cecal-ligation and puncture (CLP) bacterial challenge while 6 also included a Candida albicans challenge 3-4 days after CLP. In these six experiments $(n=322)$, CPIs were directed at the fungal challenge when CLP lethality had resolved, and were consistently beneficial $[2.91(2.41,3.50), P=0 \%, p=0.99]$. In the three experiments $(n=66)$ providing antibiotics without fungal challenge, CPIs were administered within 1 day of CLP and had variable and non-significant effects $[0.05(0.00,1.03) ; 7.86(0.28$, 217.11); and $8.50(0.90,80.03)]$. No experiment examined pneumonia.
\end{abstract}

Conclusions: Preclinical studies showing that CPIs add benefit to antibiotic therapy for the common bacterial infections causing sepsis clinically are needed to support this therapeutic approach. Studies should be reproducible across multiple laboratories and include procedures to reduce the risk of bias.

Keywords: Checkpoint molecule, Checkpoint inhibitor, Sepsis, Preclinical model, Bacterial infection, Treatment 


\section{Background}

Immune checkpoint molecules regulate $\mathrm{T}$ lymphocyte function [1] and when activated, reduce $\mathrm{T}$ cell proliferation, inflammatory cytokine production, and longevity [2, 3]. Checkpoint inhibitors (CPIs) can sustain lymphocyte activation and benefit host defense in select clinical contexts. Monoclonal antibodies (mAb) blocking programmed cell death-1 (PD-1), its ligand PD-L1, or cytotoxic T lymphocyte-associated protein-4 (CTLA-4) augment tumor-reactive cytotoxic $\mathrm{T}$ cell function and are currently FDA-approved treatments for several cancers $[4,5]$. Inhibition of PD-1 and PD-L1 has also been shown to improve pathogen clearance in some viral and protozoal infection models [6].

Checkpoint molecule expression is reportedly increased in septic patients, and there has been interest in using CPIs to augment host defense for sepsis due to acute bacterial infection $[7,8]$. This therapeutic approach has risks though, since CPIs could elicit host inflammation and aggravate sepsis-associated inflammatory injury [7]. Despite such risks, CPIs have been reported to improve bacterial clearance and survival in several animal bacterial sepsis models [9-12]. Based in part on these preclinical studies, two phase I clinical trials have been conducted testing CPI therapy in patients presenting with severe sepsis or septic shock $[13,14]$. However in one, treatment with an antiPD-L1 mAb did not have apparent benefit, and a planned phase II trial was not conducted [13]. A second phase I sepsis trial of an anti-PD-1 mAb was completed in January 2018, but further clinical trials have not been announced [14].

While these preclinical and clinical experiences with CPIs appear at odds, the sepsis field has been characterized by immunomodulator agents that were reportedly beneficial in early animal studies but failed in subsequent clinical trials. Several factors have been cited to explain these differing results. While the site and type of infection are uniform for subjects in animal studies, these vary in patients. An immunomodulator beneficial under one set of conditions might be ineffective or even harmful under another [15-17]. Also, while antibiotics are standard clinically, they are frequently not included in animal sepsis models where their absence might favor immunomodulator agents, especially ones augmenting host defense [18]. The cardiopulmonary support patients receive, which could also negate an immunomodulator's benefit, is also rarely used in animal models $[18,19]$. Finally, lack of sample size calculations and randomization and blinding procedures standard in clinical trials and the tendency to publish positive but not negative results may bias preclinical reports [20].

We sought to better understand whether factors like those cited above may have contributed to discrepant results between published animal studies and the present small clinical experience with CPIs in sepsis. We performed a systematic review and meta-analysis of published preclinical sepsis studies comparing survival in bacteria- or lipopolysaccharide (LPS)-challenged groups receiving CPIs versus control. We hypothesized that overall published animal sepsis studies would report benefit with CPI, but would not account for variables potentially influencing these agents' purported benefits.

\section{Methods}

This systematic review was registered with PROSPERO on October 15, 2018 (CRD42018109798), and prepared using the Preferred Reporting Items for Systematic Reviews and Meta-Analyses (PRISMA) statement for literature review and data extraction (Additional file 1: Appendix 1). 


\section{Literature search and study selection}

Using guidelines [21] and search strategies presented in Additional file 1: Appendix 2, two authors (LMB, PTP) identified relevant studies in PubMed, EMBASE, Scopus, and Web of Science from inception through March 13, 2019, without language restrictions. Included studies were searched for additional references. Studies were analyzed if they included experiments comparing the effect of an inhibitor of PD-1, PD-L1, CTLA-4, or BTLA to a control agent on survival following a bacterial or LPS challenge. Studies employing a non-bacterial challenge in addition to bacterial or LPS challenge were included.

\section{Data extracted and outcomes examined}

Study data were extracted by two investigators (LMB, PQE) using a standardized tool including animal number, species, strain, age, and sex; type and site of bacterial or LPS challenge; type and timing of additional challenges if used; type and regimen of CPI investigated; type and regimen of antimicrobial or other supportive treatments; and observation duration. The day of bacterial challenge was designated day 0 (D0), and other interventions were recorded relative to D0.

The primary outcome examined was the effect of CPI treatment on the odds ratio (95\% $\mathrm{CI}$ ) of survival (OR) based on the number of animals reported living at the end of observation periods. In studies including more than one experiment, each experiment was analyzed individually. In studies employing a nonspecific antibody or other protein treatment in one control group and a saline or other diluent in another, the former control group was analyzed. When survival was only provided with Kaplan-Meier plots, study authors were contacted to obtain animal numbers contributing to the plotted outcomes. If the authors did not respond, survival rates were estimated from the Kaplan-Meier plots independently by two authors (LMB, PQE) and agreed to by consensus.

Secondary outcomes assessed included the effect of infectious challenge on the checkpoint molecule targeted and the effect of CPI on bacterial counts, organ injury, cytokine levels, cytokine production by isolated cells tested ex vivo, immune cell numbers in blood or tissues, and apoptosis. Data were extracted from experiments that employed similar challenges and treatment regimens as in survival experiments and only if statistical analysis comparing CPI and control groups was provided.

Risk of bias was assessed in studies based on a modified version of the Systemic Review Centre for Laboratory Animal Experimentation (SYRCLE) grading system and whether studies reported sample size calculations; randomization of challenges and treatments; blinding of challenges, treatments, and survival assessment; confirmation of the baseline similarity of study group animals (e.g., weight, age); removal of animals during study; and randomized housing $[22,23]$.

\section{Statistical analysis}

The odds ratio of survival with CPI versus the control group was estimated using a random-effects model [24] and the Knapp-Hartung adjustment for small study numbers [25]. The effects of CPI treatment on survival were prospectively planned to be analyzed based on four variables including the checkpoint molecule targeted; site and type of bacterial challenge; and inclusion of anti-bacterial treatment. In one study in 
which CPI therapy was administered $1 \mathrm{~h}$ before or 1 or $3 \mathrm{~h}$ after LPS challenge in three different treatment groups, these groups were combined and compared to the single control group [26]. In one study [11], a common control group was used for two experiments. We split the control group data evenly so they are not used twice in our analysis. Heterogeneity among studies was assessed using the $Q$-statistic and $I^{2}$ value and was considered moderate or greater for $I^{2} \geq 35 \%$ [27]. Due to the differing assays and tissues sampled across studies for secondary outcome data, these results were summarized and presented as qualitative differences between CPI versus control groups (i.e., statistically significantly increased or decreased or not significantly different). Publication bias was assessed by funnel plot and Egger's regression [28]. All analyses were performed using $\mathrm{R}$ (version 3.6.0) packages meta (version 4.9-5) and metafor (version 2.1-0) [29-31]. Two-sided $p$ values $\leq 0.05$ were considered significant.

\section{Results}

\section{Summary of studies and experiments analyzed}

Of 1565 retrieved reports, 11 studies with 19 experiments met the inclusion criteria (Additional file 1: Figure S1) [11, 12, 26, 32-39]. These experiments were all conducted in mice and were analyzed individually. Tables 1 and 2 summarize for each experiment the type and timing of CPI therapy, the bacterial and non-bacterial challenges administered, whether and how antibacterial or other treatments were employed, and the numbers of total animals and survivors. Overall, the 19 experiments included 338 control and 371 CPI-treated animals. Importantly, of the 19 included experiments, 10 were published from the same laboratory. Additionally, assessment for risk of bias revealed that nearly all of the domains included in the SYRCLE tool were not reported, except for one study which did report blinding to treatment (Table 3).

Checkpoint inhibitors were directed against PD-L1 in nine experiments, PD-1 in three, CTLA-4 in five, and BTLA in two (referred to subsequently as anti-PD-1, antiPD-L1, anti-CTLA-4, and anti-BTLA when required). All studies employed mAbs, except for one study testing a peptide inhibitor of PD-L1 [37]. Several studies have suggested that the mAb clone used to target BTLA in the two experiments analyzed, while antagonistic under some conditions, can be agonistic under others [40-43]. All treatments will be referred to as CPIs in the text and figures.

Fifteen experiments (79\%) included cecal-ligation and puncture (CLP) bacterial challenge (i.e., polymicrobial), either alone $(n=9)$ or followed 3 to 4 days later by intravenous Candida albicans challenge $(n=6)$. One CLP model included hemorrhage challenge 1 day before CLP. Of the four experiments not employing CLP, one administered Listeria monocytogenes intravenously (IV), one LPS IP, and two either Pseudomonas aeruginosa intradermally (ID) or Staphylococcus aureus IV 4 days after skin burn. No experiment examined bacterial pneumonia.

In experiments with bacterial (including CLP) or LPS challenge alone, CPI treatment was administered starting 1 day before, on the day of, or 1 day after challenge (D-1, D0, or D+1 in Table 2). In all six experiments with CLP followed 3 to 4 days later by $C$. albicans, CPI treatment was started 1 to 2 days following fungal challenge (i.e., 4 to 6 days after CLP) and targeted the fungal infection since CLP mortality is largely resolved in 3 to 4 days. 


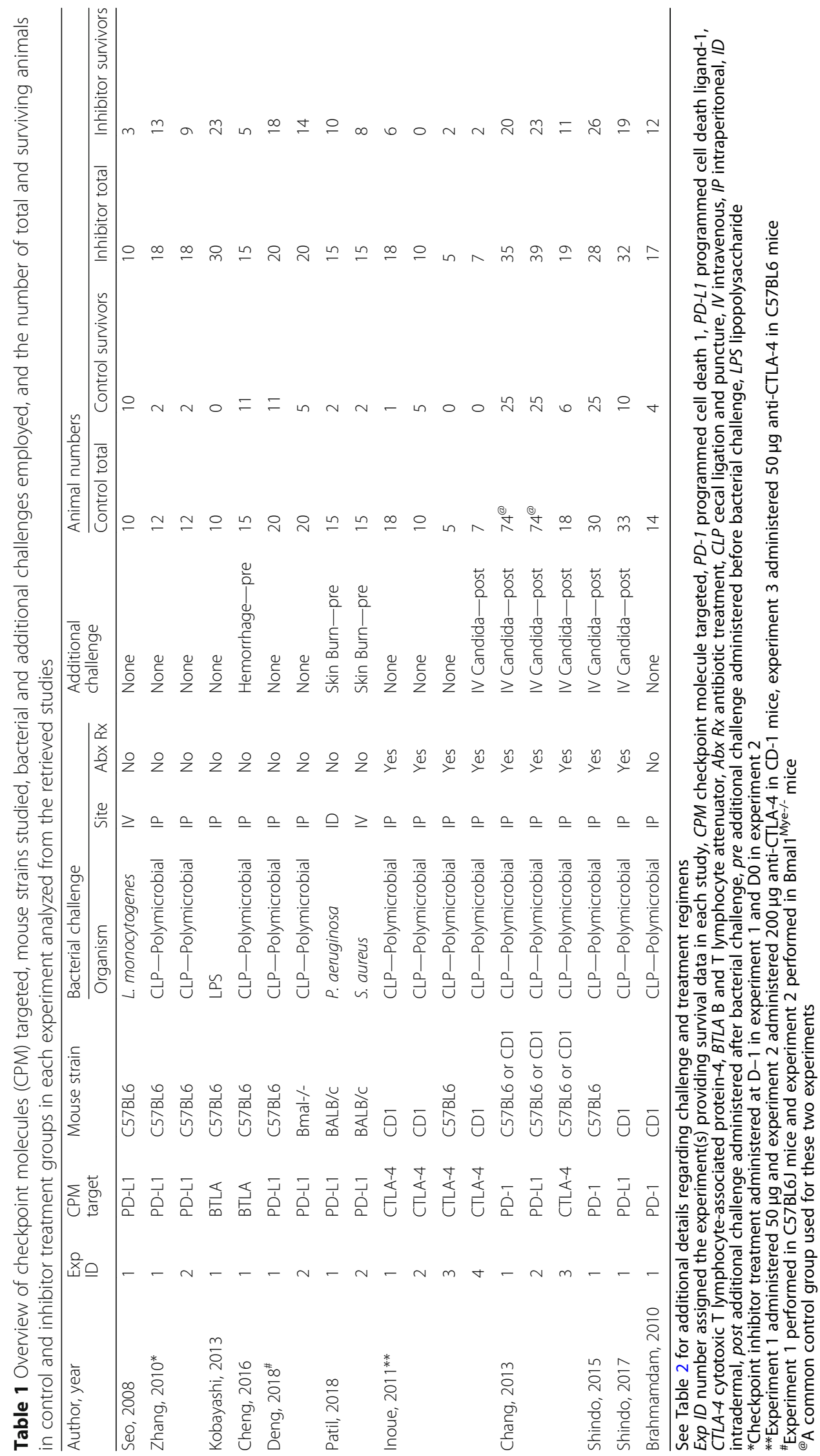




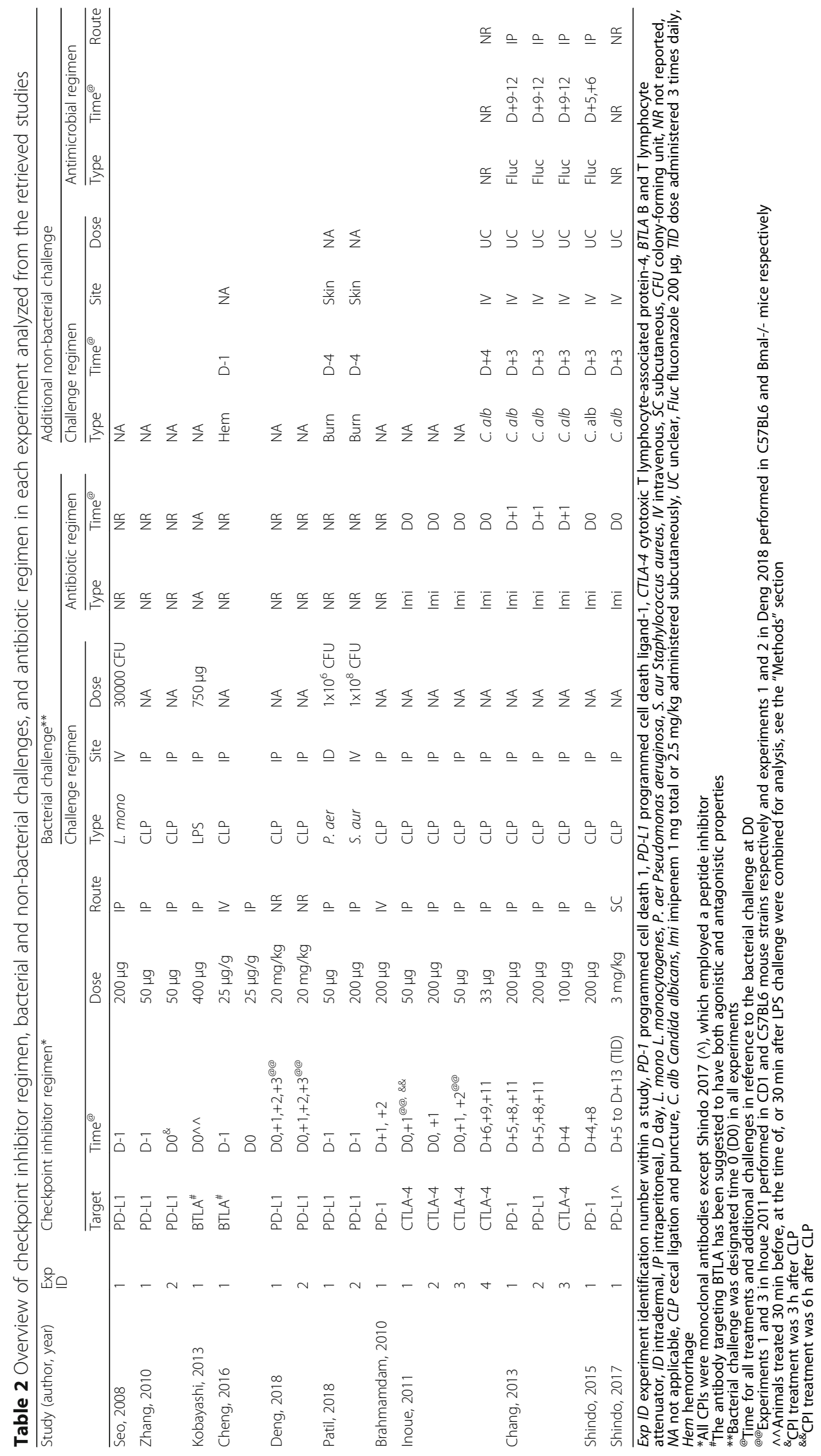




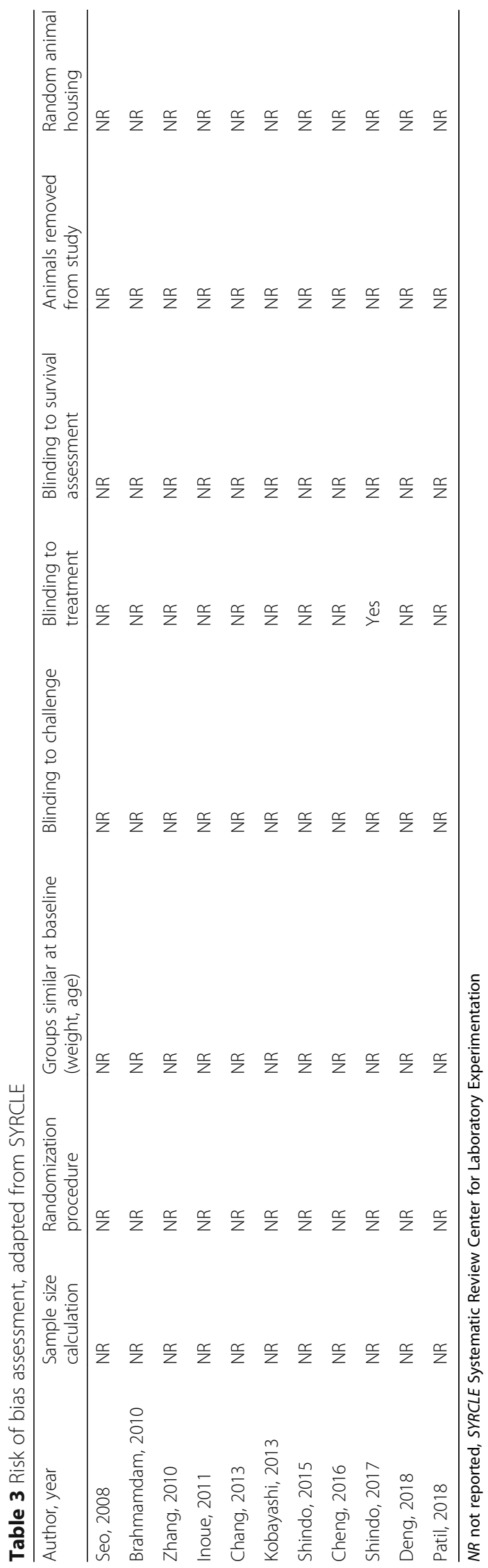


Nine experiments investigated CPI with antibacterial therapy, and these all included CLP challenge with imipenem treatment. Six of the CLP experiments also included subsequent $C$. albicans challenge, and four of these administered fluconazole with fungal challenge. While all CLP experiments included a 1- to 2-ml subcutaneous normal or phosphate-buffered saline injection following CLP, none included later hemodynamic support. The four non-CLP experiments did not include supportive measures.

\section{Effect of infectious challenge on the checkpoint molecule targeted in experiments}

Twelve experiments from eight studies (Additional file 1: Table S1) examined the effect of bacterial challenge on expression of the checkpoint molecule targeted with CPI therapy in infected versus noninfected untreated animals. In at least 11 experiments, the bacterial challenge increased the expression of the checkpoint molecule targeted $(p$ $\leq 0.05$ ). One study reported increased expression, but a $p$ value was not provided.

\section{Effect of checkpoint inhibitor therapy on survival}

CPIs increased the odds ratio of survival [OR $(95 \% \mathrm{CI})$ ] in 16 experiments $(10$ significantly) and decreased it in 3 (2 significantly) (Fig. 1). The overall OR was increased with

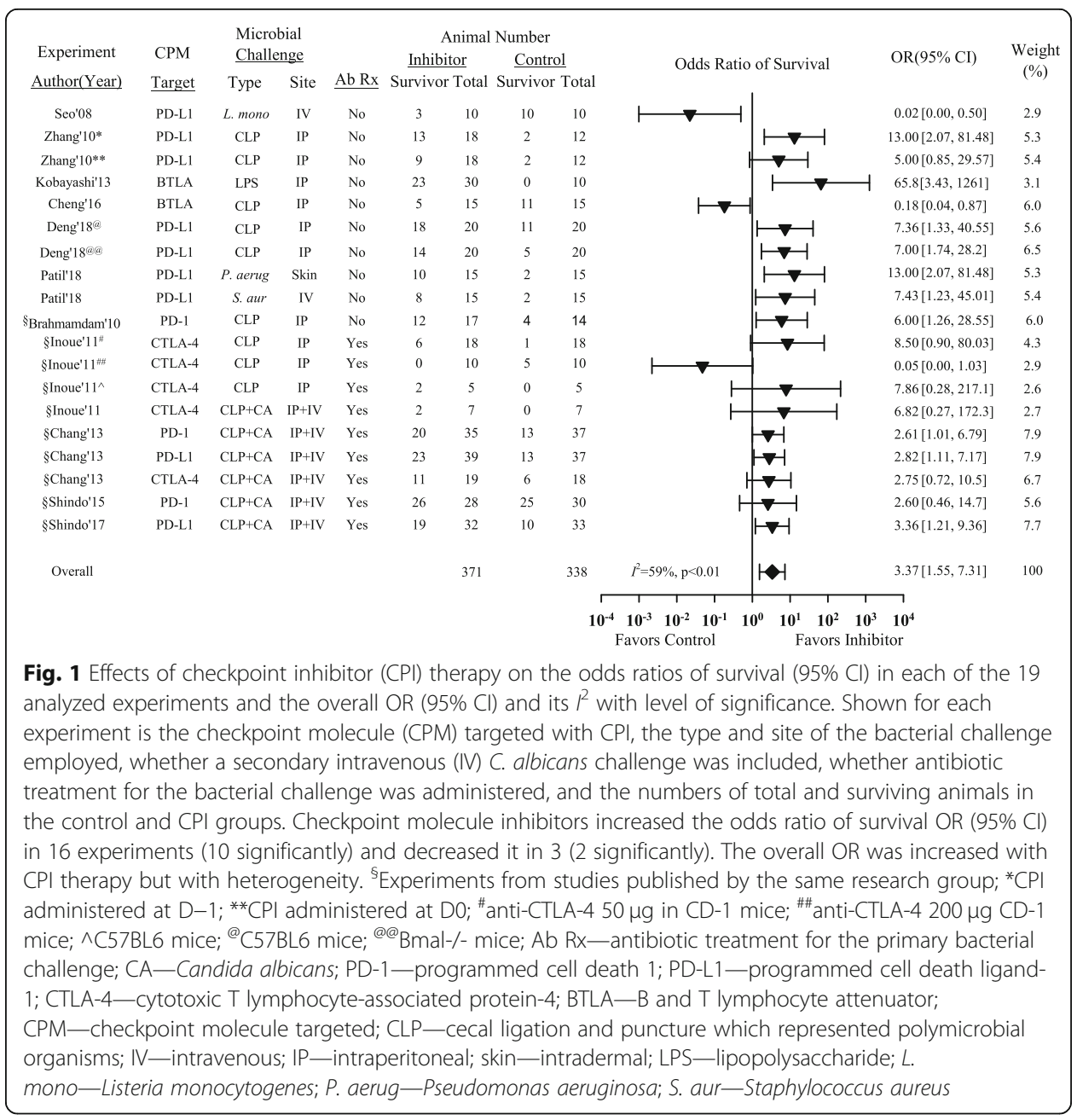


CPI therapy $[3.37(1.55,7.31)]$ but with heterogeneity $\left(I^{2}=59 \%, p<0.01\right)$. The three experiments in which CPI treatment had an effect on the side of harm included treatment with a PD-L1 mAb with IV L. monocytogenes, a high dose of CTLA-4 mAb with CLP alone, and a BTLA mAb with CLP following hemorrhage challenge.

When experiments were stratified by the type of checkpoint molecule targeted, site or type of infectious challenge, or presence of antibacterial treatment, CPI treatment had consistent effects over the greatest number of studies in the nine that included antibacterial treatment (Fig. 2). In the 10 experiments ( $n=321$ total animals) without antibacterial therapy, CPIs increased the OR in eight (seven significantly) in a pattern approaching significance but with heterogeneity $\left[4.01(0.89,18.05) ; I^{2}=74 \%, p<0.01\right]$ (Fig. 3). In the nine experiments with antibacterial therapy ( $n=388$ total animals), all with CLP challenge, CPI increased overall survival significantly and consistently [2.82 (1.60, 4.98); $I^{2}=6 \%, p=0.39$ ] (Fig. 2). However, these nine experiments only included three with CLP alone ( $n=66$ total animals) but six with CLP followed 3 to 4 days later by $C$. albicans challenge ( $n=322$ total animals). While CPI therapy was administered within 1 day of CLP in the former three experiments, it was administered following the fungal challenge in the six other experiments when lethality related to CLP would have largely resolved (i.e., 3 to 4 days after CLP); therefore, these two groups of experiments were also analyzed separately (Fig. 3). CPI administration after the fungal challenge had highly consistent beneficial effects [2.91 (2.41, 3.50); $\left.I^{2}=0 \%, p=0.99\right]$. However, in the only three experiments in this analysis which tested CPI and antibacterial therapy together with bacterial challenge alone, CPIs had highly variable effects on the ORs

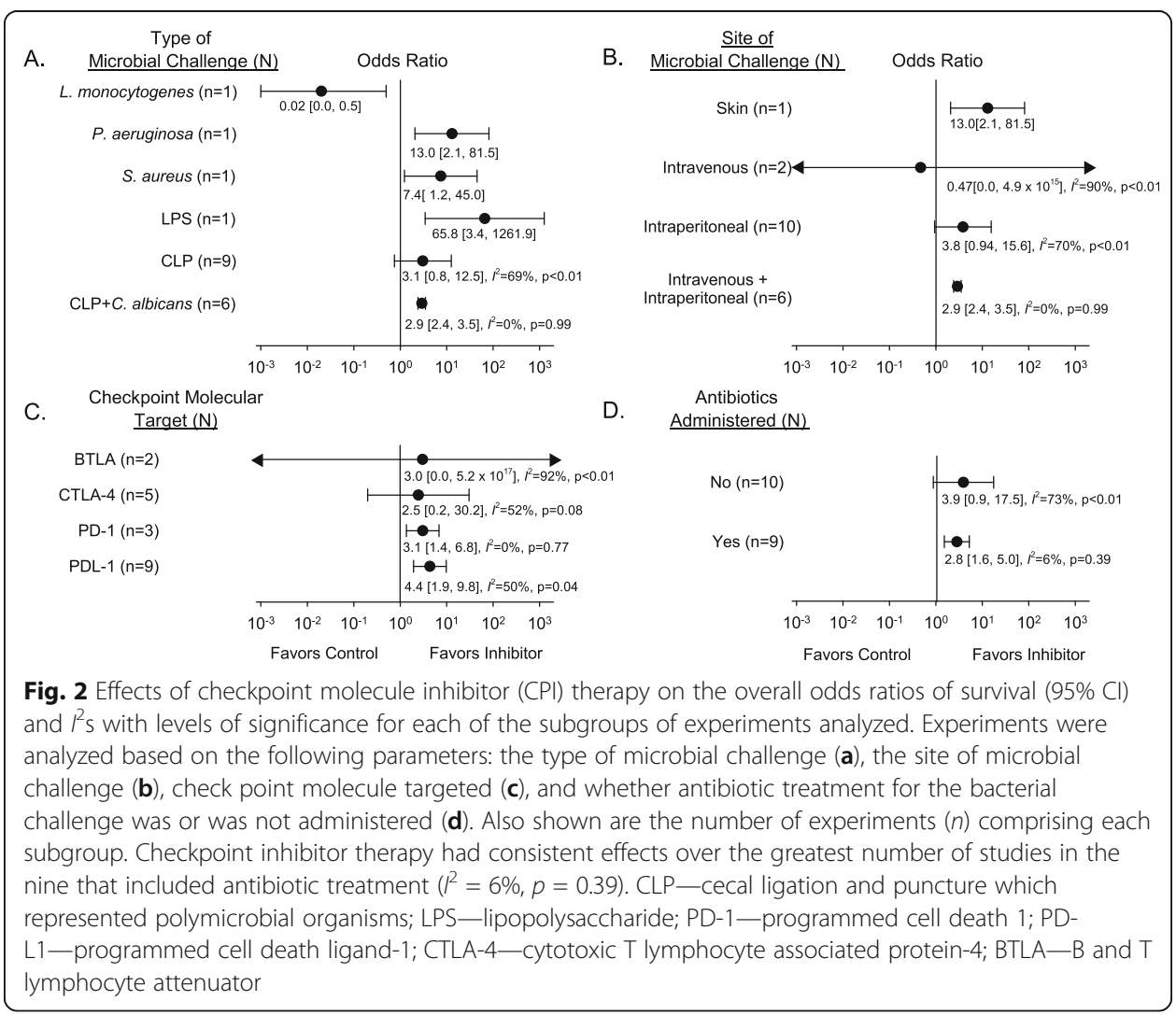




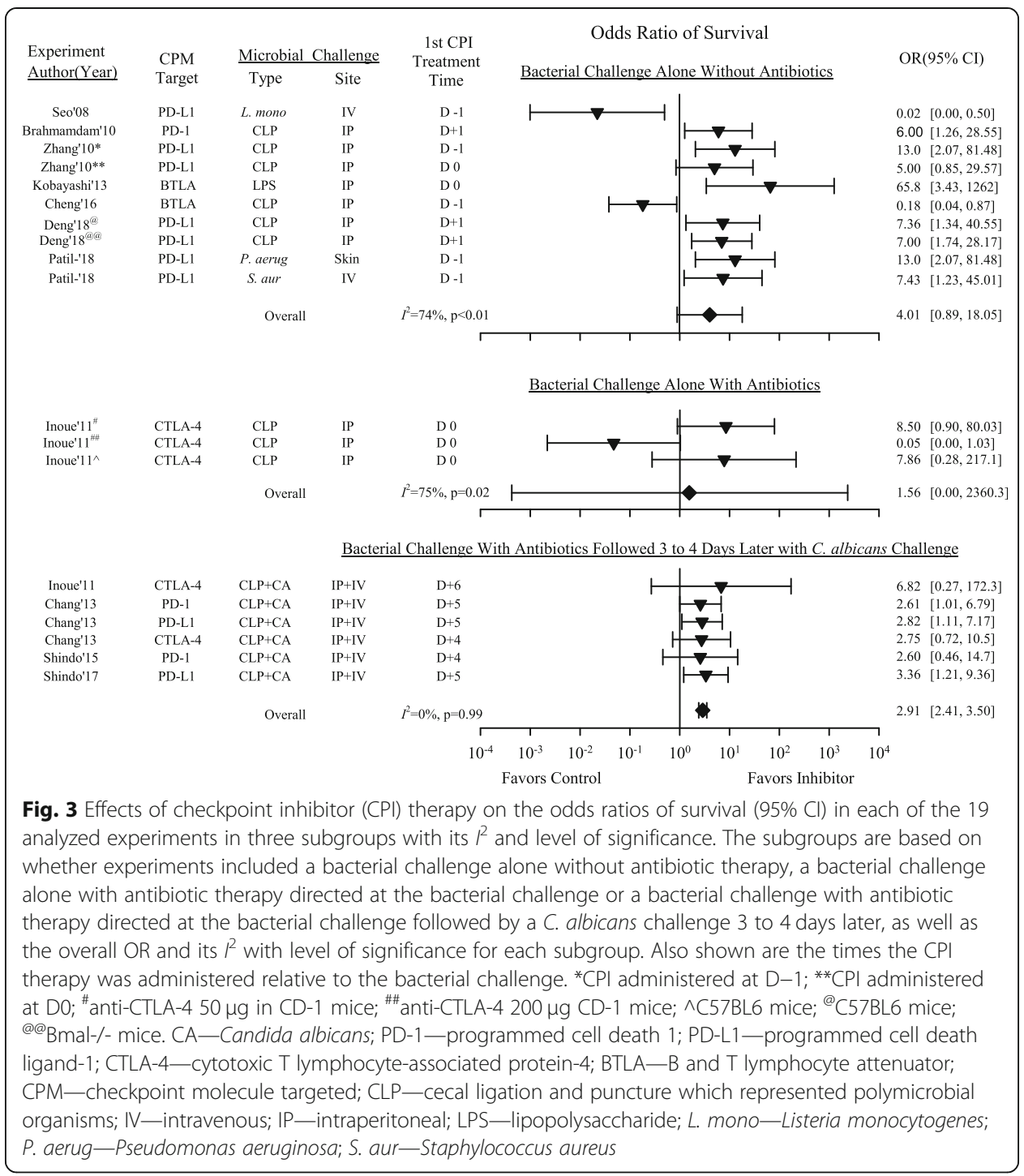

[0.05 $(0.00,1.03) ; 7.86(0.28,217.11)$; and $8.5(0.90,80.03)]$, and the overall survival effect was far from significant [1.56 $\left.(0.00,2360.31) ; I^{2}=75 \%, p=0.02\right]$.

Funnel plot and Egger's statistic $(p=0.96)$ suggested that the overall survival results were not subject to publication bias (Additional file 1: Figure S2). However, none of the 11 studies reported sample size calculations, random allocation of animals to challenges, treatments, or housing or blinding of survival assessment. Only 1 study reported blinding of treatment. Overall risk of bias was unclear in all studies (Table 3).

\section{Effect of checkpoint inhibitor treatment on microbial clearance}

As summarized in Table 4, seven experiments reported the effects of CPI treatment on quantitative bacterial cultures. None of the seven included antibiotic therapy. In three CLP experiments and one with ID P. aeruginosa challenge, anti-PD-L1 decreased bacterial counts in blood, peritoneal fluid, or lung tissue on $\mathrm{D}+1, \mathrm{D}+2$, or $\mathrm{D}+3(p \leq 0.05)$. 


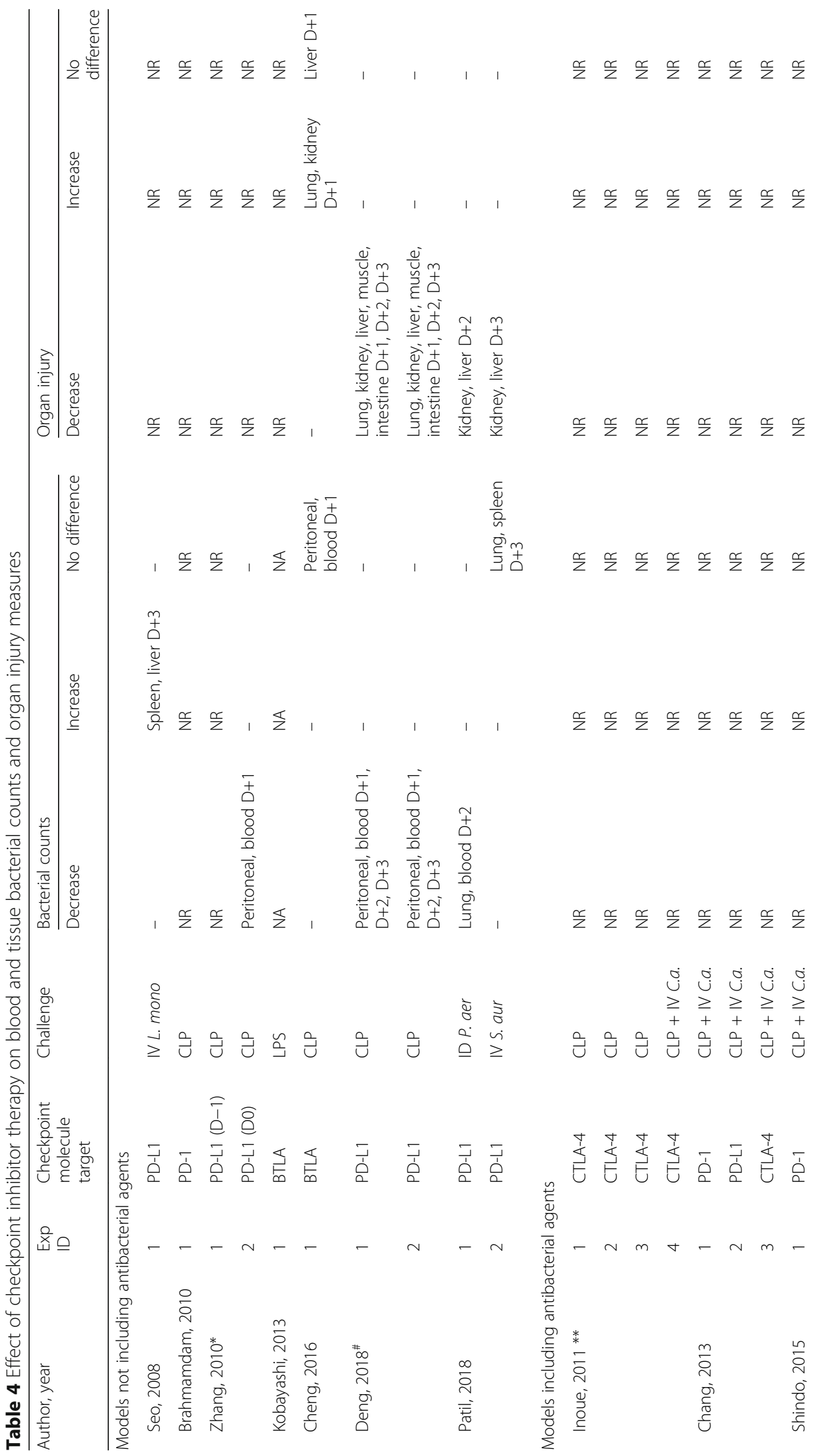




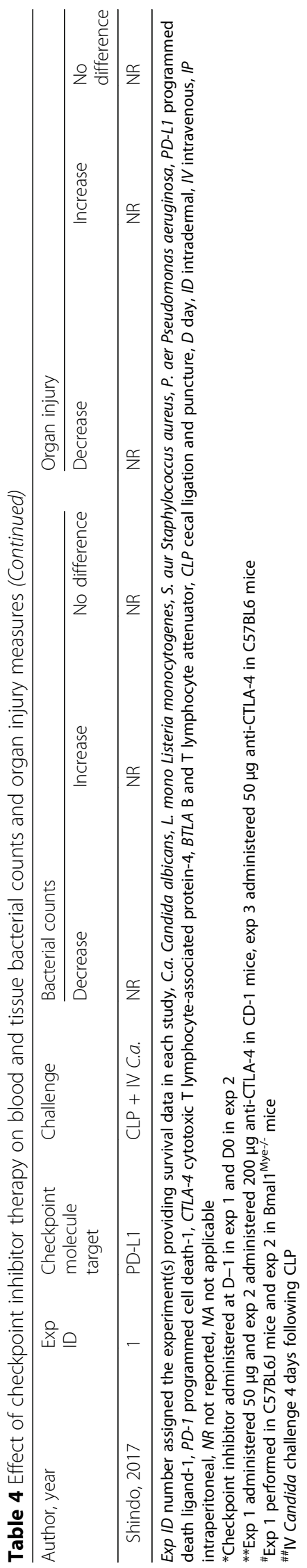


Anti-BTLA had no significant effect $(p=n s)$ on blood and peritoneal bacterial counts in one CLP experiment, and anti-PD-L1 had no significant effect on lung or spleen bacterial counts with IV $S$. aureus in another. Finally, anti-PD-L1 increased liver and spleen bacterial counts on $\mathrm{D}+3$ following IV L. monocytogenes $(p \leq 0.05)$.

\section{Effect of checkpoint inhibitor treatment on organ injury}

Five experiments, none including antibiotics, provided data regarding the effects of CPI on organ injury (Table 4). Lung, liver, renal, intestinal, and/or muscle injury as reflected by changes in lung lavage protein, serum creatinine, blood urea nitrogen levels, serum alanine or aspartate aminotransferase levels, intestinal histology, or creatine phosphokinase levels were decreased with anti-PD-L1 treatment in four experiments on D+1 to D+3 after either CLP, ID P. aeruginosa, or IV S. aureus (all $p \leq 0.05$ ). However, lung lavage protein and serum creatinine levels were increased with anti-BTLA treatment on $\mathrm{D}+1$ after CLP $(p=0.05)$.

\section{Effect of checkpoint inhibitor treatment on serum and tissue cytokines, immune cell populations, and apoptosis}

The effects of CPI treatment on serum or tissue cytokines, immune cell populations, and apoptosis were reported in five, eight, nine, and seven experiments, respectively. These results are summarized in Table 5 and described in detail in a supplemental section (Additional file 1: Appendix 3). The effects of CPI treatment on serum and tissue cytokine levels were variable, and no clear pattern was evident. However, possibly consistent with CPI treatment's proposed pro-inflammatory effects, in one study, anti-PDL1 treatment was associated with increases in TNF $\alpha$ and IL-6 levels, and a concomitant decrease in IL-10 [12]. Checkpoint inhibitor treatment increased the numbers or activation state of at least one immune cell population in seven experiments but decreased the population examined in one and had no effect in another. Finally, CPI treatment decreased immune cell apoptosis in five studies but had no effect in two.

\section{Discussion}

This systematic review suggests several reasons why CPI treatment appeared beneficial in published preclinical sepsis models but not in the present small clinical experience. It also highlights important methodological issues which must be considered when interpreting the results of these preclinical studies. The finding that over half of the experiments were published from a single laboratory raises an important question about generalizability of these results. Furthermore, all studies lacked sufficient reporting of methods designed to reduce bias, including provision of sample size calculations, randomization, and blinding procedures. The importance of these procedures is wellsupported by groups such as SYRCLE and ARRIVE. Just as in clinical trials, the extent to which reliable conclusions can be drawn from preclinical studies that are systematically reviewed and used as support for human trials is dependent upon the rigor of these studies' investigative methods [22, 44].

While the primary rationale for CPI treatment in sepsis is the augmentation of host defense and microbial clearance, there are actually little published animal data demonstrating that early CPI therapy combined with antibacterial treatment improves 


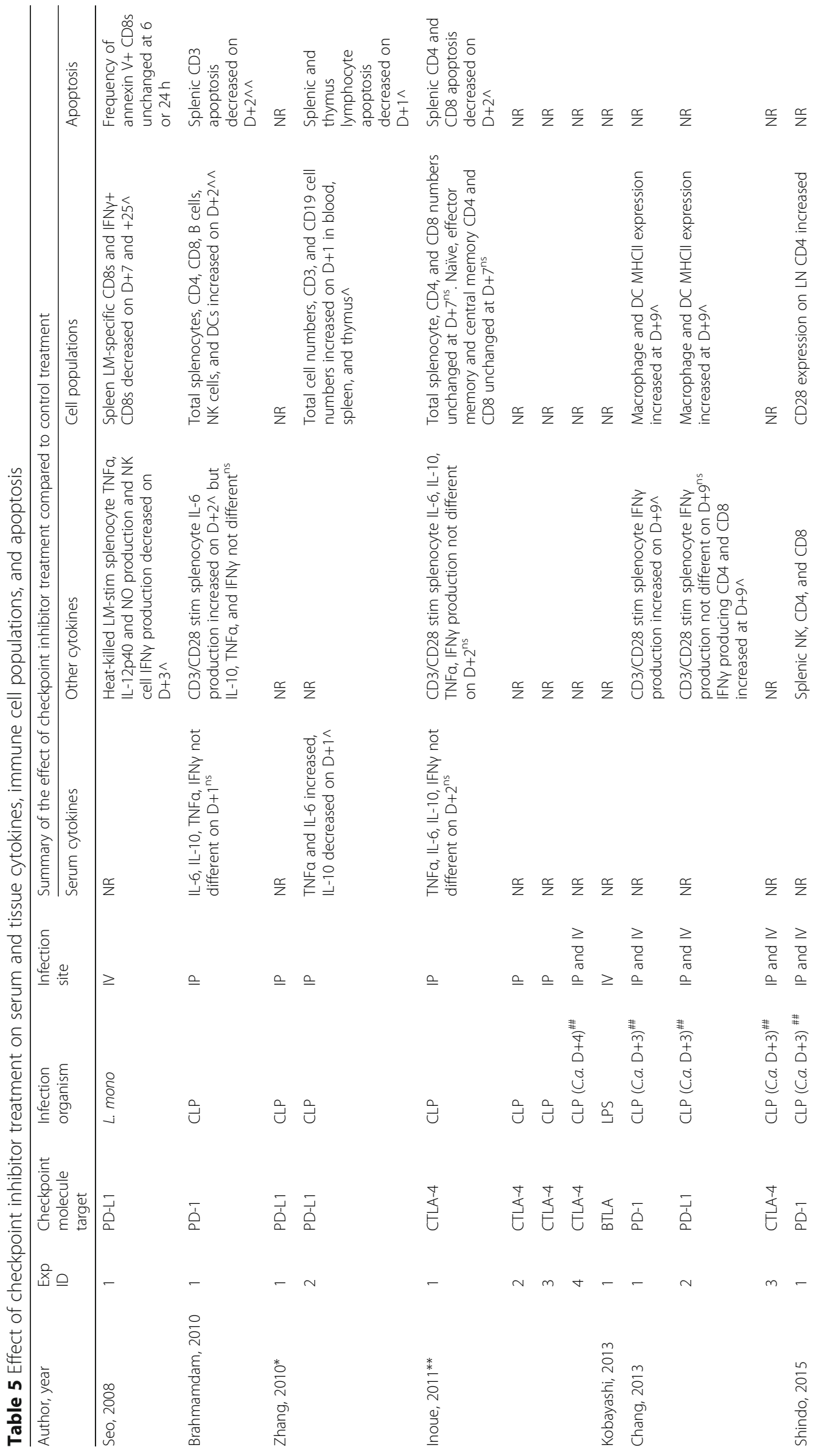




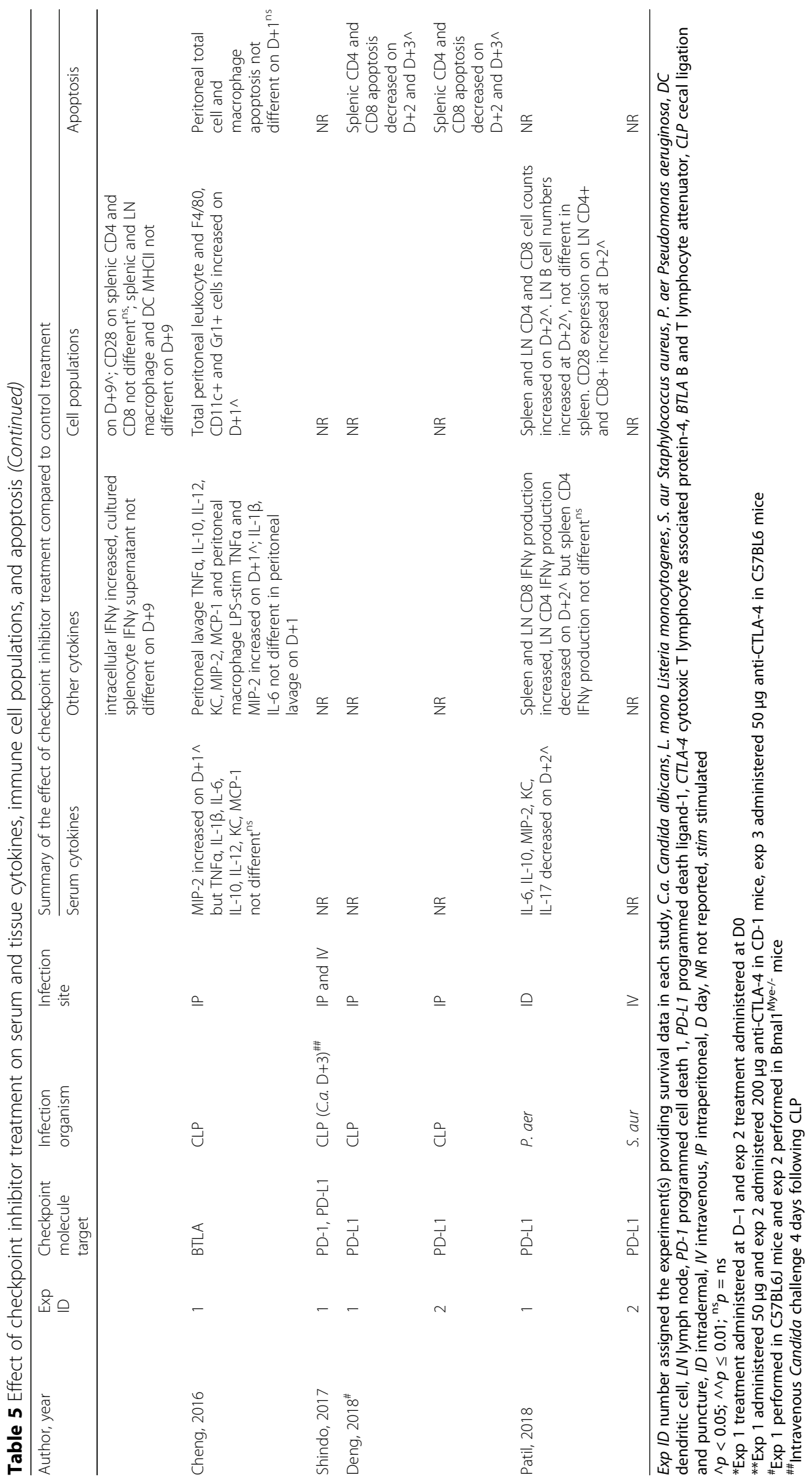


outcome following bacterial infection. In the ten experiments without antimicrobial agents, CPI treatment increased survival in a trend approaching significance. Also, in the six experiments with CLP and antibacterial therapy followed 3 to 4 days later by $C$. albicans challenge, CPI therapy targeting the fungal challenge had highly consistent beneficial effects. But in the only three experiments that investigated a bacterial challenge alone with antibacterial therapy (i.e., CLP with imipenem), CPI treatment had highly variable effects and was not significantly beneficial. Notably, these three experiments only included 66 animals and were all conducted in a single study. Additional investigation might have shown a more consistent effect.

Other factors may also explain the differing treatment effects of CPIs when comparing the overall preclinical and limited clinical experience to date. First, no published preclinical experiment investigated pneumonia, where CPI's risk of augmented inflammatory lung injury might interfere with its potential host defense benefit. Yet pulmonary infection is the most frequent cause of sepsis in medical intensive care units, as it was in the anti-PD-L1 phase I trial [13]. Second, these mouse studies did not include cardiopulmonary support comparable to what patients receive, which could negate the benefit observed with CPI treatment. Third, preclinical models do not typically reflect the variety of comorbidities prevalent in critically-ill populations. Finally, the absence of sample size calculations and randomization and blinding procedures in preclinical studies may have confounded the survival findings with CPI treatment.

When examined in the 19 experiments overall, a basis for CPIs' purported beneficial survival effect is not clear. Only seven experiments reported whether CPIs affected microbial clearance and none of these included antibacterial treatment which could negate CPIs' effects. While anti-PD-L1 did improve survival and reduce blood, peritoneal, or lung bacterial counts in four experiments, it had no effect on bacterial counts in two, and actually increased spleen and liver bacterial counts in one experiment with IV L. monocytogenes challenge. Several experiments did suggest that CPI enhanced some host defense effects including increased splenic lymphocyte numbers, IFN $\gamma$-producing CD4 cells or activated lymphocytes in five experiments, and decreased apoptosis in five.

It is also unclear whether increased survival with CPIs was related to the reduced organ injury since only five experiments provided these data. In four experiments, antiPD-L1 increased survival and decreased evidence of lung, liver, kidney, and/or intestinal injury with either CLP alone or skin $P$. aeruginosa or intravenous $S$. aureus infection after burn injury. None of these experiments included antibacterial treatment, and in three, as noted above, CPIs were associated with reduced bacterial counts. Thus, while improved host defense with CPIs may have reduced tissue injury, this effect could be negated by antibacterial agents.

Consistent improvements in survival in the six experiments with CLP in which CPIs were administered following a subsequent $C$. albicans challenge (four of which also included antifungal therapy) suggest CPIs might be beneficial with fungal superinfection. However, fungal superinfection in patients presenting with bacterial sepsis and not already severely immunosuppressed is actually uncommon. In 1719 patients presenting with bacterial sepsis, only 32 (1.9\%) developed a secondary fungal infection [45].

In three experiments, CPI treatment was associated with worsened survival that was or approached significance (Fig. 1). In one experiment with CLP following hemorrhage, anti-BTLA treatment did not alter bacterial counts but was associated with increased 
lung and kidney injury. As noted in the results, this study employed an anti-BTLA antibody with possible agonistic rather than antagonistic activity $[41,42]$. In the study with IV L. monocytogenes challenge, an anti-PD-L1 mAb decreased L. monocytogenes-specific CD8 cells and IFN $\gamma+C D 8$ cells and increased spleen and liver bacterial counts. In this case, it is also possible this CPI actually suppressed host defense [32]. In the last of these three experiments, animals challenged with CLP and treated with an antibacterial agent received a dose of anti-CTLA-4 dose four times greater than the one that was protective in another experiment from the same study. In this study, the larger dose of anti-CTLA-4 may have produced inflammatory injury not present with the lower dose, but this study did not provide data on organ injury or microbial clearance to make this assessment.

The present study has limitations. As noted, the absence of microbiologic and organ injury data in most experiments prevented understanding how CPI treatment improved survival. Also, the lack of data regarding sample size calculations, randomization, and blinding procedures prevented an accurate assessment of risk of bias in all studies. In fact, only one study reported a single blinding procedure, and no study provided methods for randomization or sample size calculations. Additionally, five of the 11 studies and all nine experiments with CLP and antibiotic treatment were conducted by a single research group. Confirmation of the findings from these experiments by other groups would be important.

\section{Conclusions}

In conclusion, the present findings suggest that if CPI therapy continues to be a consideration for early sepsis, there should be additional preclinical investigation showing that it will add benefit and not harm when used in combination with standard antibiotic and other supportive measures. These studies should include the range of bacterial infections that septic patients present with and should be conducted with procedures that limit potential risk of bias.

\section{Supplementary information}

Supplementary information accompanies this paper at https://doi.org/10.1186/s40635-019-0290-x.

Additional file 1. Appendix 1. Prisma checklist. Appendix 2. Literature search strategy. Appendix 3. Effect of checkpoint inhibitor treatment on serum and tissue cytokines, immune cell populations, and apoptosis. Table 1. Effect of bacterial challenge on the checkpoint molecules targeted in analyzed experiments. Figure 1. Flow chart of literature search and study selection. Figure 2. Funnel plot of odds ratio (OR) of survival.

\section{Abbreviations}

ARRIVE: Animal Research: Reporting of In Vivo Experiments; BTLA: B- and T-lymphocyte attenuator; Cl: Confidence interval; CLP: Cecal-ligation and puncture; CPIs: Checkpoint inhibitors; CTLA-4: Cytotoxic T-lymphocyte associated protein-4; ID: Intradermally; LPS: Lipopolysaccharide; OR: Odds ratio for survival; PD-1: Programmed death-1; PD-L1: PDligand1; PRISMA: Preferred Reporting Items for Systematic Reviews and Meta-Analyses; SYRCLE: Systemic Review Centre for Laboratory Animal Experimentation

\section{Acknowledgements}

The authors thank Judith Welsh, BSN, MLS, for her contribution in designing and performing the literature search.

\section{Author contributions}

LMB and PTP had full access to all of the data in the study and take responsibility for the integrity of the data and the accuracy of the data analysis, including and especially any adverse effects. LMB, JS, XC, PQE, and PTP contributed substantially to the study design, data analysis, and interpretation. LMB and PTP drafted the manuscript, and JS and $P Q E$ revised it critically for important intellectual content. $L M B, J S, X C, P Q E$, and PTP approved the final version to be published. 


\section{Funding}

Intramural funding from the National Institutes of Health supported this work.

\section{Availability of data and materials}

The datasets used and/or analyzed during the current study are available from the corresponding author on reasonable request.

\section{Ethics approval and consent to participate}

Not applicable

\section{Consent for publication}

Not applicable

\section{Competing interests}

The authors declare that they have no competing interests.

Received: 12 August 2019 Accepted: 27 December 2019

Published online: 04 February 2020

\section{References}

1. Attanasio J, Wherry EJ (2016) Costimulatory and coinhibitory receptor pathways in infectious disease. Immunity 44: 1052-1068

2. Buchbinder El, Desai A (2016) CTLA-4 and PD-1 pathways: similarities, differences, and implications of their inhibition American Journal of Clinical Oncology 39:98-106

3. Honda T, Egen JG, Lammermann T, Kastenmuler W, Torabi-Parizi P, Germain RN (2014) Tuning of antigen sensitivity by T cell receptor-dependent negative feedback controls T cell effector function in inflamed tissues. Immunity 40:235-247

4. Callahan MK, Postow MA, Wolchok JD (2016) Targeting T cell co-receptors for cancer therapy. Immunity 44:1069-1078

5. Chen L, Han X (2015) Anti-PD1/PD-L1 therapy of human cancer: past, present, and future. The Journal of Clinical Investigation 125:3384-3391

6. Rao M, Valentini D, Dodoo E, Zumla A, Maeurer M (2017) Anti-PD-1/PD-L1 therapy for infectious diseases: learning from the cancer paradigm. International Journal of Infectious Diseases 56:221-228

7. Cavaillon JM, Eisen D, Annane D (2014) Is boosting the immune system in sepsis appropriate? Crit Care 18:216

8. Davies R, O'Dea K, Gordon A (2018) Immune therapy in sepsis: are we ready to try again? J Intensive Care Soc 19:326-344

9. Huang X, Chen YP, Chung CS, Yuan ZL, Monaghan SF, Wang F, Ayala A (2014) Identification of B7-H1 as a novel mediator of the innate immune/proinflammatory response as well as a possible myeloid cell prognostic biomarker in sepsis. J Immunol 192:1091-1099

10. Huang X, Venet F, Wang YL, Lepape A, Yuan Z, Chen Y, Swan R, Kherouf H, Monneret G, Chung CS, Ayala A (2009) PD-1 expression by macrophages plays a pathologic role in altering microbial clearance and the innate inflammatory response to sepsis. Proc Natl Acad Sci U S A 106:6303-6308

11. Chang KC, Burnham CA, Compton SM, Rasche DP, Mazuski R, JS MD, Unsinger J, Korman AJ, Green JM, Hotchkiss RS (2013) Blockade of the negative co-stimulatory molecules PD-1 and CTLA-4 improves survival in primary and secondary fungal sepsis. Crit Care 17:R85

12. Zhang Y, Zhou Y, Lou JS, Li JB, Bo LL, Zhu KM, Wan XJ, Deng XM, Cai ZL (2010) PD-L1 blockade improves survival in experimental sepsis by inhibiting lymphocyte apoptosis and reversing monocyte dysfunction. Crit Care 14:R220

13. Hotchkiss RS, Colston E, Yende S, Angus DC, Moldawer LL, Crouser ED, Martin GS, Coopersmith CM, Brakenridge S, Mayr FB, Park PK, Ye J, Catlett IM, Girgis IG, Grasela DM (2019) Immune checkpoint inhibition in sepsis: a phase 1b randomized, placebo-controlled, single ascending dose study of antiprogrammed cell death-ligand 1 (BMS-936559). Crit Care Med 47:632-642

14. Hotchkiss RS, Colston E, Yende S, Crouser ED, Martin GS, Albertson T, Bartz RR, Brakenridge SC, Delano MJ, Park PK, Doninno MW, Tidswell M, Mayr FB, Angus DC, Coopersmith CM, Moldawer LL, Catlett IM, Girgis IG, Ye J, Grasela DM (2019) Immune checkpoint inhibition in sepsis: a Phase 1b randomized study to evaluate the safety, tolerability, pharmacokinetics, and pharmacodynamics of nivolumab. Intensive Care Med 45:1360-71.

15. Bagby GJ, Plessala KJ, Wilson LA, Thompson JJ, Nelson S (1991) Divergent efficacy of antibody to tumor necrosis factor-a in intravascular and peritonitis model of sepsis. J Inf Dis 163:83-88

16. Eichacker PQ, Parent C, Kalil A, Esposito C, Cui X, Banks SM, Gerstenberger EP, Fitz Y, Danner RL, Natanson C (2002) Risk and the efficacy of antiinflammatory agents. Am J Resp Crit Care 166:1197-1205

17. Karzai W, Cui X, Mehlhorn B, Straube E, Hartung T, Gerstenberger E, Banks SM, Natanson C, Reinhart K, Eichacker PQ (2003) Protection with antibody to tumor necrosis factor differs with similarly lethal Escherichia coli versus Staphylococcus aureus pneumonia in rats. Anesthesiology 99:81-89

18. Hellman J, Bahrami S, Boros M, Chaudry IH, Fritsch G, Gozdzik W, Inoue S, Radermacher P, Singer M, Osuchowski MF, Huber-Lang M (2019) Part III: Minimum quality threshold in preclinical sepsis studies for fluid resuscitation and antimicrobial therapy endpoints. Shock 51:33-43

19. Qui P, Li Y, Ding Y, Weng J, Banks SM, Kern S, Fitz Y, Suffredini AF, Eichacker PQ, Cui X (2011) The individual survival benefits of tumor necrosis factor soluble receptor and fluid administration are not additive in a rat sepsis model. Intensive Care Med 37:1688-1695

20. Lewis AJ, Lee JS, Rosengart MR (2018) Translational sepsis research: spanning the divide. Crit Care Med 46:1497-1505

21. Stroup DF, Berlin JA, Morton SC, Olkin I, Williamson GD, Rennie D, Moher D, Becker BJ, Sipe TA, Thacker SB (2000) Metaanalysis of observational studies in epidemiology: a proposal for reporting. Meta-analysis Of Observational Studies in Epidemiology (MOOSE) group. JAMA 283:2008-2012 
22. Hooijmans CR, Rovers MM, de Vries RB, Leenaars M, Ritskes-Hoitinga M, Langendam MW (2014) SYRCLE's risk of bias tool for animal studies. BMC Med Res Methodol 14:43

23. Wever KE, Geessink FJ, Brouwer MAE, Tillema A, Ritskes-Hoitinga M (2017) A systematic review of discomfort due to toe or ear clipping in laboratory rodents. Lab Anim 51:583-600

24. DerSimonian R, Laird N (1986) Meta-analysis in clinical trials. Control Clin Trials 7:177-188

25. Knapp G, Hartung J (2003) Improved tests for a random effects meta-regression with a single covariate. Stat Med 22: 2693-2710

26. Kobayashi Y, Iwata A, Suzuki K, Suto A, Kawashima S, Saito Y, Owada T, Kobayashi N, Nakajima H (2013) B and T lymphocyte attenuator inhibits LPS-induced endotoxic shock by suppressing Toll-like receptor 4 signaling in innate immune cells. Proc Natl Acad Sci U S A 110:5121-5126

27. Higgins JP, Thompson SG (2002) Quantifying heterogeneity in a meta-analysis. Stat Med 21:1539-1558

28. Egger M, Davey Smith G, Schneider M, Minder C (1997) Bias in meta-analysis detected by a simple, graphical test. BMJ 315:629-634

29. Core Team R. R Foundation for Statistical Computing. 2004; https:/www.r-project.org/. Accessed May 10, 2018.

30. Schwarzer G. meta: General package for meta-analysis. R package version. 2015; https://CRAN.R-project.org/package= meta. Accessed May 10, 2018

31. Viechtbauer W (2010) Conducting meta-analyses in R with the metafor package. J Stat Softw 36:1-48

32. Seo SK, Jeong HY, Park SG, Lee SW, Choi IW, Chen L, Choi I (2008) Blockade of endogenous B7-H1 suppresses antibacterial protection after primary Listeria monocytogenes infection. Immunology 123:90-99

33. Brahmamdam P, Inoue S, Unsinger J, Chang KC, McDunn JE, Hotchkiss RS (2010) Delayed administration of anti-PD-1 antibody reverses immune dysfunction and improves survival during sepsis. J Leukoc Biol 88:233-240

34. Inoue S, Bo L, Bian J, Unsinger J, Chang K, Hotchkiss RS (2011) Dose-dependent effect of anti-CTLA-4 on survival in sepsis. Shock 36:38-44

35. Shindo Y, Unsinger J, Burnham CA, Green JM, Hotchkiss RS (2015) Interleukin-7 and anti-programmed cell death 1 antibody have differing effects to reverse sepsis-induced immunosuppression. Shock 43:334-343

36. Cheng T, Bai J, Chung CS, Chen Y, Biron BM, Ayala A (2016) Enhanced innate inflammation induced by anti-BTLA antibody in dual insult model of hemorrhagic shock/sepsis. Shock 45(1):40-49.

37. Shindo Y, McDonough JS, Chang KC, Ramachandra M, Sasikumar PG, Hotchkiss RS (2017) Anti-PD-L1 peptide improves survival in sepsis. J Surg Res 208:33-39

38. Deng W, Zhue S, Zeng L, Liu J, Kang R, Yang M, Cao L, Wang H, Billiar TR, Jiang J, Xie M, Tang D (2018) The circadian clock controls immune checkpoint pathway in sepsis. Cell Reports 24:366-378

39. Patil NK, Luan L, Bohannon JK, Hernandez A, Guo Y, Sherwood ER (2018) Anti-PD-L1 protects against infection with common bacterial pathogens after burn injury. J Leukoc Biol 103:23-33

40. Albring JC, Sandau MM, Rapaport AS, Edelson BT, Satpathy A, Mashayekhi M, Lathrop SK, Hsieh CS, Stelljes M, Colonna M, Murphy TL, Murphy KM (2010) Targeting of B and T lymphocyte associated (BTLA) prevents graft-versus-host disease without global immunosuppression. J Exp Med 207:2551-2559

41. Hurchla MA, Sedy JR, Gavrielli M, Drake CG, Murphy TL, Murphy KM (2005) B and T lymphocyte attenuator exhibits structural expression polymorphisms and is highly induced in anergic CD4+ T cells. J Immunol 174:3377-3385

42. Lepenies B, Pfeffer K, Hurchla MA, Murphy TL, Murphy KM, Oetzel J, Fleischer B, Jacobs T (2007) Ligation of B and T lymphocyte attenuator prevents the genesis of experimental cerebral malaria. J Immunol 179:4093-4100

43. Tao R, Wang L, Han R, Wang T, Ye Q, Honjo T, Murphy TL, Murphy KM, Hancock WW (2005) Differential effects of B and T lymphocyte attenuator and programmed death-1 on acceptance of partially versus fully MHC-mismatched cardiac allografts. J Immunol 175:5774-5782

44. Kilkenny C, Browne WJ, Cuthill IC, Emerson M, Altman DG (2010) Improving bioscience research reporting: the ARRIVE guidelines for reporting animal research. PLoS biology 8:e1000412-e1000412

45. van Vught LA, Klein Klouwenberg PMC, Spitoni C, Scicluna BP, Wiewel MA, Horn J, Schultz MJ, Nurnberg P, Bonten MJM, Cremer OL, van der Poll T (2016) Incidence, risk factors, and attributable mortality of secondary infections in the intensive care unit after admission for sepsis. JAMA 315:1469-1479

\section{Publisher's Note}

Springer Nature remains neutral with regard to jurisdictional claims in published maps and institutional affiliations.

\section{Submit your manuscript to a SpringerOpen ${ }^{\circ}$ journal and benefit from:}

- Convenient online submission

- Rigorous peer review

- Open access: articles freely available online

- High visibility within the field

- Retaining the copyright to your article

Submit your next manuscript at $\boldsymbol{\nabla}$ springeropen.com 